\title{
Call-Burst Blocking Probabilities of ON-OFF Traffic Sources under the Bandwidth Reservation Policy
}

\author{
Ioannis D. Moscholios and Michael D. Logothetis \\ University of Patras, Dept. of Electrical \& Computer Engineering, \\ Wire Communications Laboratory, 26500 Patras, Greece \\ \{moscholios, m-logo\} @wcl.ee.upatras.gr
}

\begin{abstract}
We calculate call and burst blocking probabilities (CBP and BBP respectively) in a single link loss system accommodating service-classes of $\mathrm{ON}$ OFF traffic calls, either of high or low bandwidth requirements. To benefit high speed calls the bandwidth reservation (BR) policy is considered (ON-OFF/BR model). Poisson arriving calls enter the system via state $\mathrm{ON}$ and may alternate between states ON and OFF. When a call passes to state OFF it releases the bandwidth held in state $\mathrm{ON}$ which becomes available for new arrivals. When the call tries to return to state $\mathrm{ON}$ it re-requests its bandwidth. If it is available a new burst begins; otherwise, the burst is blocked and the call remains in state OFF. The CBP/BBP calculation is based on approximate formulas.
\end{abstract}

\section{Introduction}

Two bandwidth sharing policies are the most common at call-level: the complete sharing (CS) and the bandwidth reservation (BR) policy [1]. When calls of different service-classes compete for the available bandwidth of a link, under the CS policy (i.e. calls equally share the link bandwidth upon arrival, without any restriction), this leads to an unfair bandwidth allocation among the service-classes. Service-classes with higher bandwidth per call requirements will receive worse call-level QoS, namely, call blocking probability (CBP), than other service-classes accommodated in the link with fewer requirements. Usually an equal CBP among the service-classes is desirable; this is achieved with the BR policy. According to the BR policy, some fraction of the free bandwidth of the link is reserved to benefit the high speed calls.

We consider the ON-OFF model of [2] under the BR policy (ON-OFF/BR) and focus on the $\mathrm{CBP}$ equalization among the service-classes. According to the $\mathrm{BR}$ policy, the CBP equalization is achieved when the reserved link bandwidth for a service-class is defined so that the sum of it and the required bandwidth per call of the service-class is the same for all service-classes. The absence of a product form solution (PFS) [3] in the $\mathrm{ON}-\mathrm{OFF} / \mathrm{BR}$ model motivates the necessity for an approximate CBP calculation. To this end, we rely on the recursive formula of [2] and propose a similar-structured formula that achieves satisfactory CBP results. For the BBP calculation, we propose an approximate formula, which stands not only for the BR but also for the CS policy. For the derivation of this formula we follow the assumption that the population of calls of a service-class inside its reservation space (RS) is negligible [1]. The resultant $\mathrm{BBP}$ formula is based on the calculation of the average number of calls in the burst 
blocking state space. This calculation (and consequently the BBP) is improved by the method of Stasiak\&Glabowski (S\&G) [4] that takes into account the average population of calls inside the service-class RS that it is not negligible in reality.

In section 2 we propose the ON-OFF/BR model. We give an approximate but recursive CBP formula and derive a BBP formula. On the latter we apply the method of $S \& G$ in order to obtain better BBP results. In section 3 we present numerical and simulation CBP/BBP results for evaluation. We conclude in section 4.

\section{The Proposed ON-OFF Model under the BR Policy}

Consider a single link of capacity $C$, accommodating $K$ service-classes of ON-OFFtype calls. Calls of a service-class $k(k=1, \ldots, K)$ arrive to the link according to a Poisson process with mean arrival rate $\lambda_{k}(\mathrm{calls} / \mathrm{sec})$ and require $b_{k}$ bandwidth units (b.u). The available link bandwidth is shared according to the CS policy. A call is accepted in the link in state ON, while the occupied link bandwidth is characterized real - real link. At the end of the ON-period a call of service-class $k$ releases the b.u that holds and may begin an OFF-period with probability $\sigma_{k}$, or depart from the system with probability $1-\sigma_{k}$. While it is in state OFF, it is assumed that it seizes fictitious bandwidth $\left(b_{k}\right)$ of a fictitious link with fictitious capacity $C^{*}$. At the end of the OFF-period a call of service-class $k$ returns to state $\mathrm{ON}$ with probability 1 . However, in order to return to state $\mathrm{ON}$ it requires again $b_{k}$ b.u. If $C=C^{*}$, there is always available bandwidth for that call in state ON, i.e. no burst blocking occurs. If $C<C^{*}$, then if there is available bandwidth in the real link, i.e. if $j_{1}+b_{k} \leq C$, (where $j_{1}$ is the occupied real link bandwidth), the call will return to state $\mathrm{ON}$ and a new burst will begin; otherwise the burst is blocked and the call will remain in state OFF for another OFF-period. When the BR policy is incorporated to the ON-OFF model, a new service-class $k$ call is accepted in the system with $b_{k}$ b.u., if it meets the following constraints:

$$
\begin{gathered}
j_{1}+b_{k} \leq C-t_{k} \\
j_{1}+j_{2}+b_{k} \leq C^{*}-t_{k}
\end{gathered}
$$

where $t_{k}$ is the BR parameter of service-class $k$, and $j_{2}$ is the occupied fictitious link bandwidth.

The second constraint prevents the system from accepting new calls when most of the system calls are in state OFF. This model does not have a PFS; therefore the calculation of $G(\vec{j})$ 's, is done in an approximate way, according to the recursive formula:

$$
\begin{gathered}
j_{s} G(\vec{j})=\sum_{i=1}^{2} \sum_{k=1}^{K} D_{i, k, s}\left(\vec{j}-B_{i, k}\right) p_{i k} G\left(\vec{j}-B_{i, k}\right) \\
\text { where: } D_{i, k, s}\left(\vec{j}-B_{i, k}\right)= \begin{cases}b_{i, k, s} & \text { when } j_{1} \leq C-t_{k} \text { and } j_{2}+j_{1} \leq C^{*}-t k \\
0 & \text { otherwise }\end{cases}
\end{gathered}
$$

where, $i=1 \Rightarrow$ state ON, $i=2 \Rightarrow$ state OFF and $s=1 \Rightarrow$ real link, $s=2 \Rightarrow$ fictitious link. 


$$
b_{i, k, s}= \begin{cases}b_{k} & \text { if } s=i \\ 0 & \text { if } s \neq i\end{cases}
$$

$B_{i, k}$ : is the $(i, k)^{\text {th }}$ row of the $(2 K \times 2)$ matrix $B$ with entries $b_{i, k, s}$ and $B_{i, k}=\left(b_{i, k, 1} b_{i, k, 2}\right)$; $p_{i k}$ : the utilization of the $i^{\text {th }}$ type link by service-class $k$,

$$
p_{i k}=\frac{e_{i k}}{\mu_{i k}}= \begin{cases}\frac{\lambda_{k}}{\left(1-\sigma_{\kappa}\right) \mu_{I \kappa}} & \text { for } i=1 \\ \frac{\lambda_{k} \sigma_{\kappa}}{\left(1-\sigma_{\kappa}\right) \mu_{2 \kappa}} & \text { for } i=2\end{cases}
$$

$e_{i k}, \mu_{i k}^{1}:$ total arrival rate and mean service time of service-class $k$ calls in $i^{\text {th }}$ state.

The CBP of a service-class $k$, denoted as $P_{b}$, is calculated by:

$$
P_{b_{k}}=\underset{\left\{\vec{j} \mid\left[\left(b_{1, k, 1}+j_{1}\right)>C-t_{k}\right] \cup\left[\left(b_{1, k, 1}+j_{1}+j_{2}\right)>C^{*}-t_{k}\right]\right\}}{\sum G^{-1} G(\vec{j})} \text { where } G=\sum_{\vec{j} \in \Omega} G(\vec{j})
$$

The BBP of a service-class $k$, denoted as $P_{b_{k}}^{*}$, is calculated by:

$$
P_{b_{k}}^{*}=\frac{\sum_{\left(\overrightarrow{j \in \Omega^{*}}\right)} y_{2 k}(\vec{j}) G(\vec{j}) \mu_{2 k}}{\sum_{(\vec{j} \in \Omega)} y_{2 k}(\vec{j}) G(\vec{j}) \mu_{2 k}}
$$

where $\vec{j} \in \Leftrightarrow\left\{\left(j_{1} \leq C \cap\left(\sum_{s=1}^{2} j_{s} \leq C^{*}\right)\right)\right\}, y_{2 k}(\vec{j})$, the average population of calls of service-class $\mathrm{k}$ in state OFF (when the system state is $\vec{j}$ ) determined by ([5]):

$$
y_{i k}(\vec{j})=E\left(n_{k}^{i} \mid \vec{j}\right)=\frac{p_{i k} G\left(\vec{j}-B_{i, k}\right)}{G(\vec{j})}, i=1,2
$$

and the $\mathrm{G}(\vec{j})$ 's are calculated by (3).

To prove (7), if $\bar{n}_{k}^{i}$ is the average number of service-class k calls in $i^{\text {th }}$ state ([2]):

$$
\bar{n}_{k}^{i}=\sum_{(\overrightarrow{j \in \Omega})} p_{i k} G\left(\vec{j}-B_{i, k}\right)
$$

note the relation between (8) and (9):

$$
\bar{n}_{k}^{i}=\sum_{(\overrightarrow{j \in \Omega})} y_{i k}(\vec{j}) G(\vec{j})
$$

Multiplying $y_{2 k}(\vec{j})$ by the corresponding $G(\vec{j})$ and the service rate in state OFF $\mu_{2 k}$ and summing up over the burst blocking space: 


$$
\vec{j} \in \Omega^{*} \Leftrightarrow\left\{\left(C-b_{k}+1 \leq j_{1} \leq C \cap\left(\sum_{s=1}^{2} j_{s} \leq C^{*}\right)\right)\right\}
$$

we obtain the following summation:

$$
\sum_{\left(\overrightarrow{\left.j \in \Omega^{*}\right)}\right.} y_{2 k}(\vec{j}) G(\vec{j}) \mu_{2 k}
$$

By normalizing it (taking into account the whole state space ), we obtain (7).

The application of the BR policy to the ON-OFF model leads to $y_{2 k}(\vec{j})=0$ when $j_{1}$ $>C-t_{k}$ or $j_{2}>C^{*}-j_{1}-t_{k}$. We have investigated that, depending on the values of $t_{k}$, equation (7) may result in a poor approximation of the BBP. A better approximation is obtained when one applies in (7) the method of S\&G for the $y_{2 k}(\vec{j})$ calculation, according to the following proposed procedure:

Calculation of BBP of a service-class $k$ with $t_{k}>0$

For the system state $\vec{j}=\left(j_{1}, j_{2}\right)$

If $j_{1} \leq c-t_{k}$ and $j_{2} \leq C^{*}-j_{1}-t_{k}$ then

Determine $\mathrm{y}_{2 \mathrm{k}}(\vec{j})$ according to Eq (8) and Eq. (3) .

endif

If $j_{\text {real }}>C-t_{k}$ then

For all service-classes $\mathrm{x}(\mathrm{x}=1, \ldots, \mathrm{K}$, but $\mathrm{x} \neq \mathrm{k})$

If $j_{1} \leq C-t_{x}$ and $j_{2} \leq C^{*}-j_{1}-t_{x}$ then

$\mathrm{Y}_{2 \mathrm{k}}(\vec{j})=\mathrm{Y}_{2 \mathrm{k}}(\vec{j})+\mathrm{Y}_{2 \mathrm{k}}\left(j_{1}-b_{x}, j_{2}\right)$

endif

endif

Determine the BBP according to Eq. (7)

\section{Numerical Examples - Evaluation}

Consider a link of (real) capacity $\mathrm{C}=80$ that accommodates three service-classes. The traffic description parameters of the service-classes are the following: $\left(\lambda_{1}, \lambda_{2}, \lambda_{3}\right)=$ (0.05, 0.06, 0.09), $\left(\mu_{11}^{1}, \mu_{12}^{1}, \mu_{13}^{1}\right)=(0.5,1.0,1.2),\left(\mu_{21}^{1}, \mu_{22}^{1}, \mu_{23}^{1}\right)=(0.8,1.9,0.9),\left(b_{1}, b_{2}\right.$, $\left.b_{3}\right)=(10,8,6),\left(\sigma_{1}, \sigma_{2}, \sigma_{3}\right)=(0.85,0.9,0.95)$. CBP equalization is achieved by using the BR parameters: $\left(t_{1}, t_{2}, t_{3}\right)=(0,2,4)$. Two cases are examined: a) $\mathrm{C}=\mathrm{C}^{*}=80$ and b) $\mathrm{C}=80, \mathrm{C}^{*}=100$. In case (a) no burst blocking occurs, while in case (b) the increase of the fictitious capacity results in the burst blocking occurrence for all service-classes. 
Each point in the horizontal axis of the figures entitled "arrival rate" has the following value: point 1 is $\left(\lambda_{1}, \lambda_{2}, \lambda_{3}\right)=(0.05,0.06,0.09)$, point 2 is $\left(\lambda_{1}, \lambda_{2}, \lambda_{3}\right)=(0.05$, $0.06,0.12), \ldots$, point 6 is $\left(\lambda_{1}, \lambda_{2}, \lambda_{3}\right)=(0.05,0.06,0.24)$. Fig. 1 presents for the case (a), the analytical equalized CBP of all service-classes together with the analytical CBP of each service-class when the BR policy is not applied. As it was expected from the equalization of the CBP, Fig. 1 shows that the BR policy benefits the $1^{\text {st }}$ and the $2^{\text {nd }}$ service-class. Fig. 2 presents the analytical CBP for the case (b) for all service-classes either when the BR policy is applied or not. Simulation CBP results found to be quite close to the analytical CBP results for both cases and therefore they are not presented. The increase of the fictitious capacity from 80 to 100 decreases the CBP probabilities (more calls pass to state OFF, releasing bandwidth in state $\mathrm{ON}$ which is seized by new arrivals); the CBP of Fig. 2 are much lower than those of Fig. 1.

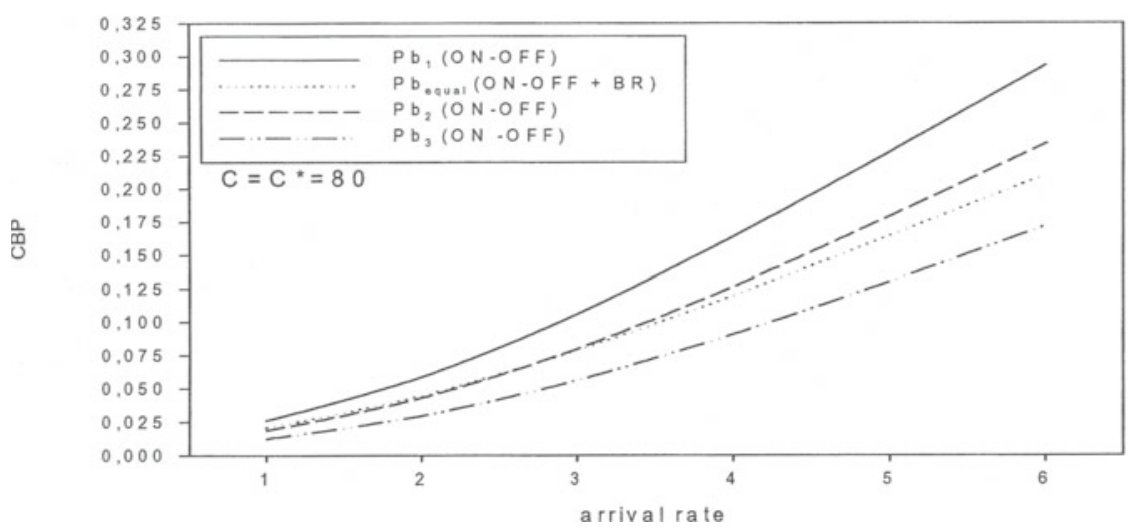

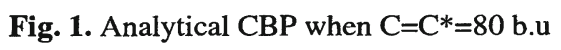

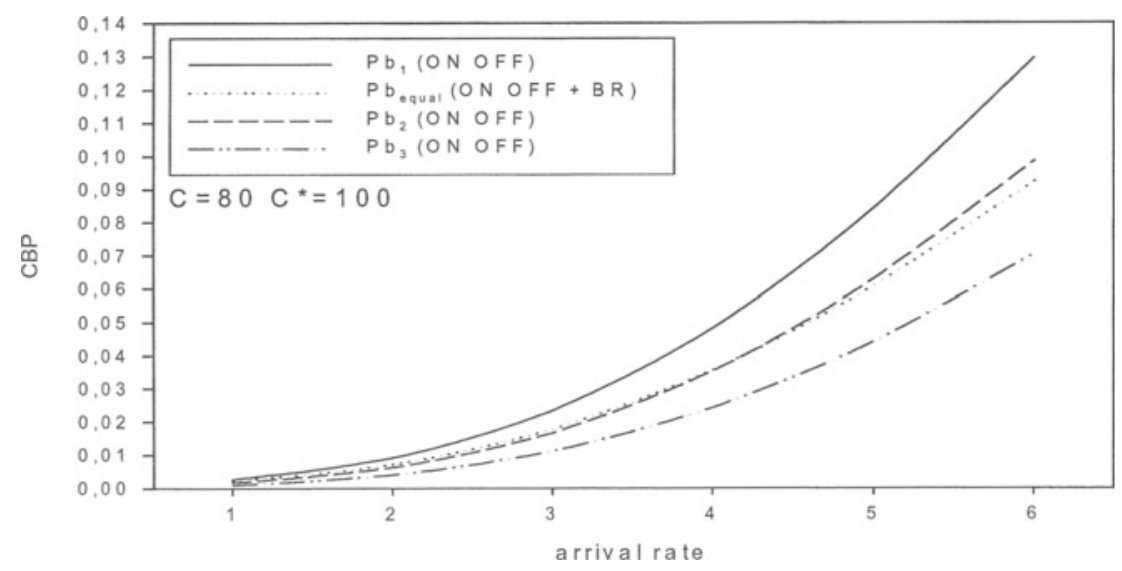

Fig. 2. Analytical $\mathrm{CBP}$ when $\mathrm{C}=80$ and $\mathrm{C}^{*}=100$ b.u.

The increase of the fictitious capacity results in the appearance of BBP for all service-classes. In Table 1 we give both analytical and simulation BBP of all serviceclasses under the BR policy. Simulation results are mean values of 10 runs with $95 \%$ 
confidence interval. The analytical BBP results of the $2^{\text {nd }}$ and $3^{\text {rd }}$ service-class are obtained by either the application of the method of $S \& G$ or not. For the $1^{\text {st }}$ service-class, however, the application of the method of S\&G in the BBP formula does not affect the BBP results, since there is no RS for the $1^{\text {st }}$ service-class $\left(t_{1}=0\right)$. For the $2^{\text {nd }}$ serviceclass, the method of $S \& G$ results in slightly better BBP results compared to the corresponding results when the method of $S \& G$ is not applied. For the $3^{\text {rd }}$ service-class, the method of $S \& G$ results in much better BBP results compared to the corresponding results when the method of $S \& G$ is not applied.

Table 1. Analytical and simulation BBP of the ON-OFF model under the BR policy

\begin{tabular}{|c|c|c|c|c|c|c|c|c|}
\hline \multirow[b]{2}{*}{$\mathrm{P}$} & \multicolumn{2}{|c|}{$\mathrm{P}_{\mathrm{b}_{1}}^{*}$} & \multicolumn{3}{|c|}{$\mathrm{P}^{*} \mathrm{~b}_{2}$} & \multicolumn{3}{|c|}{$\mathrm{P}_{\mathrm{b}_{3}}$} \\
\hline & Analytical & Simulation & Analytical & Anal.S\&G & Simulation & Analytical & Anal.S\&G & Simulation \\
\hline 1 & $9.06 \times 10^{-5}$ & $\begin{array}{c}9.59 \times 10^{-5} \\
\pm 1.45 \times 10^{-5} \\
\end{array}$ & $4.67 \times 10^{-5}$ & $4.69 \times 10^{-5}$ & $\begin{array}{c}5.62 \times 10^{-5} \\
\pm 8.90 \times 10^{-6}\end{array}$ & $2.00 \times 10^{-5}$ & $2.66 \times 10^{-5}$ & $\begin{array}{c}3.06 \times 10^{-5} \\
\pm 7.05 \times 10^{-6} \\
\end{array}$ \\
\hline 2 & $2.87 \times 10^{-4}$ & $\begin{array}{c}2.77 \times 10^{-4} \\
\pm 4.90 \times 10^{-5} \\
\end{array}$ & $1.48 \times 10^{-4}$ & $1.49 \times 10^{-4}$ & $\begin{array}{c}1.58 \times 10^{-4} \\
\pm 3.22 \times 10^{-5}\end{array}$ & $6.44 \times 10^{-5}$ & $8.33 \times 10^{-5}$ & $\begin{array}{c}9.24 \times 10^{-5} \\
\pm 9.05 \times 10^{-6}\end{array}$ \\
\hline 3 & $6.86 \times 10^{-4}$ & \begin{tabular}{|c|}
$6.35 \times 10^{-4}$ \\
$\pm 8.51 \times 10^{-5}$ \\
\end{tabular} & $3.54 \times 10^{-4}$ & 3.56 & $\begin{array}{c}3.71 \times 10^{-4} \\
\pm 3.80 \times 10^{-5} \\
\end{array}$ & $1.56 \times 10^{-4}$ & 1.9 & $\begin{array}{c}2.14 \times 10^{-4} \\
\pm 1.35 \times 10^{-5} \\
\end{array}$ \\
\hline 4 & $1.34 \times 10^{-3}$ & $\begin{array}{r}1.15 \times 10^{-3} \\
\pm 1.32 \times 10^{-4} \\
\end{array}$ & $6.83 \times 10^{-4}$ & $6.87 \times 10^{-4}$ & \begin{tabular}{|}
$7.48 \times 10^{-4}$ \\
$\pm 6.10 \times 10^{-5}$ \\
\end{tabular} & $3.01 \times 10^{-4}$ & $3.71 \times 10^{-4}$ & \begin{tabular}{|c|}
$4.35 \times 10^{-4}$ \\
$\pm 1.89 \times 10^{-5}$ \\
\end{tabular} \\
\hline 5 & $2.25 \times 10^{-3}$ & \begin{tabular}{|c|}
$2.07 \times 10^{-3}$ \\
$\pm 1.16 \times 10^{-4}$ \\
\end{tabular} & $1.12 \times 10^{-3}$ & 1.12 & $\begin{array}{c}1.27 \times 10^{-3} \\
\pm 9.00 \times 10^{-5}\end{array}$ & $4.87 \times 10^{-4}$ & $10^{-4}$ & $\begin{array}{c}7.68 \times 10^{-4} \\
\pm 3.46 \times 10^{-5} \\
\end{array}$ \\
\hline 6 & $3.38 \times 10^{-3}$ & $\begin{array}{c}2.98 \times 10^{-3} \\
\pm 1.52 \times 10^{-4} \\
\end{array}$ & $1.61 \times 10^{-3}$ & $1.62 \times 10^{-3}$ & $\begin{array}{c}1.77 \times 10^{-3} \\
\pm 8.61 \times 10^{-5}\end{array}$ & $6.91 \times 10^{-4}$ & $8.25 \times 10^{-4}$ & $\begin{array}{c}9.58 \times 10^{-4} \\
\pm 4.40 \times 10^{-5}\end{array}$ \\
\hline
\end{tabular}

\section{Conclusion}

We investigate the call and burst level behavior of service-classes of Poisson arriving ON-OFF-type calls to a single link, under the BR policy. We propose the ON$\mathrm{OFF} / \mathrm{BR}$ model (recursive $\mathrm{CBP}$ and robust BBP formulas). The accuracy of the analytical CBP and BBP results is satisfactory taken as standard simulation results.

\section{References}

1. J.W. Roberts, "Teletraffic models for the Telecom 1 Integrated Services Network", ITC-10, 1983.

2. M. Mehmet Ali, "Call-burst blocking and call admission control in a broadband network with bursty sources", Performance Evaluation, 38 (1999), pp. 1-19.

3. K. W. Ross, "Multiservice Loss Models for Broadband Telecommunications Networks", Springer Verlag London Limited, ISBN 3-540-19918-7, 1995.

4. M. Stasiak, M. Glabowski, "A simple approximation of the link model with reservation by a one-dimensional Markov chain", Performance Evaluation 41 (2000), pp. 195-208.

5. I. Moscholios, P. Nikolaropoulos, M. Logothetis, "Call level blocking of ON-OFF traffic sources with retrials under the complete sharing policy", ITC-18, Berlin, 2003, pp. 811-821. 